\title{
THE HILBERT SERIES OF MEASURES OF ENTANGLEMENT FOR 4 QUBITS
}

\author{
NOLAN R. WALLACH
}

\begin{abstract}
We determine the Hilbert series of measures of entanglement for 4 qubits. Various techniques of constructive invariant theory are applied to prove the formula.
\end{abstract}

\section{INTRODUCTION}

In most quantum computing frameworks the basic objects are superpositions of bit strings of a fixed length, $n$. These bit strings form an orthonormal basis of a Hilbert space of dimension $N=2^{n}$. The basis elements are usually labeled $\left|a_{n-1} a_{n-2} \cdots a_{0}\right\rangle$ with $a_{i} \in\{0,1\}$ following a variant of Dirac's notation. This also avoids the confusion between a basis element and the coefficients in the superposition. If $0 \leq j<N$ then if $a_{n-1} a_{n-2} \cdots a_{0}$ is the base two expression for $j$ (expanded by 0's, if necessary, to make it a bit string of length $n$ ) then the corresponding basis element is also denoted $|j\rangle$. The most basic transformation of a bit string is the operation of NOT on one of its elements that is the flip $0 \rightarrow 1,1 \rightarrow 0$. We can look upon NOT as a unitary transformation of our space of superpositions. The group generated by the flips in all possible bits is of order $2^{n}$ (isomorphic with the product $\mathbb{Z}_{2}^{n}$ ) and acts simply transitively on the set of all bit strings. In quantum computing the simplest transformations form a much larger group which we will now explain.

If $n=1$ then our Hilbert space has basis $|0\rangle,|1\rangle$ the pertinent elements are the unit vectors

$$
a|0\rangle+b|1\rangle
$$

with $|a|^{2}+|b|^{2}=1$. Two such vectors are considered to be the same if they differ by multiplication by a complex number of absolute value 1 (in other words the states of interest are elements of one dimensional projective space over $\mathbb{C}$ ). Such a class is called a qubit. It is considered to be in the state $|0\rangle$ with probability $|a|^{2}$ and in the state $|1\rangle$ with probability $|b|^{2}$. If $n=2$ then we have as orthonormal basis $|00\rangle,|01\rangle,|10\rangle,|11\rangle$ our 2 qubit states are unit vectors

$$
a|00\rangle+b|01\rangle+c|10\rangle+d|11\rangle
$$

with the same identification. That is elements of 3 dimensional projective space. We note that we can look at this 4 dimensional space of 2 qubits as the tensor product of two copies of 1 qubit space. By considering $\left|a_{1} a_{0}\right\rangle$ as $\left|a_{1}\right\rangle \otimes\left|a_{0}\right\rangle$. With

Date: August 13, .2003.

2000 Mathematics Subject Classification. Primary 05C38, 15A15; Secondary 05A15, 15A18.

Key words and phrases. Keyword one, keyword two, keyword three. 
this identification we have the operation of NOT in the first position is

$$
\left[\begin{array}{ll}
0 & 1 \\
1 & 0
\end{array}\right] \otimes\left[\begin{array}{ll}
1 & 0 \\
0 & 1
\end{array}\right] .
$$

The group generated by the operations of NOT in the two positions therefore gives rise to the action of $\mathbb{Z}_{2}^{2}$ on $\mathbb{C}^{2} \otimes \mathbb{C}^{2}$ with

$$
\begin{aligned}
& (1,0) \longmapsto\left[\begin{array}{ll}
0 & 1 \\
1 & 0
\end{array}\right] \otimes\left[\begin{array}{ll}
1 & 0 \\
0 & 1
\end{array}\right], \\
& (0,1) \longmapsto\left[\begin{array}{ll}
1 & 0 \\
0 & 1
\end{array}\right] \otimes\left[\begin{array}{ll}
0 & 1 \\
1 & 0
\end{array}\right]
\end{aligned}
$$

and $u+v \longmapsto S T$ if $u \longmapsto S, v \longmapsto T$ (here the addition is component wise mod 2 ). For arbitrary $n$ we do the same. That is, the space of $n$ qubits is projective space of dimension $N-1$. The Hilbert space is just $H_{n}=\mathbb{C}^{2} \otimes \mathbb{C}^{2} \otimes \cdots \otimes \mathbb{C}^{2}$ $n$-fold product. The action of the group generated by NOT in each factor is the group of all transformations $J_{0} \otimes J_{1} \otimes \cdots \otimes J_{n-1}$ with

$$
J_{i} \in\left\{\left[\begin{array}{ll}
0 & 1 \\
1 & 0
\end{array}\right],\left[\begin{array}{ll}
1 & 0 \\
0 & 1
\end{array}\right]\right\} .
$$

The analogous quantum transformations of qubits are of the same form except one allows $J_{i} \in U(2)$ the $2 \times 2$ unitary group. Such transformations are called local. We note that the group of local transformations acting on $H_{n}$ is up to multiplication by scalars of absolute value 1 given by the subgroup where the $J_{i} \in S U(2)$ (unitary transformations of determinant 1). We will take our basic group to be the group, $G$, consisting of the transformations as above with the $J_{i} \in S U(2)$.

It is an oversimplification to say that the local transformations are all easily implemented. However, there are enough that are that we can take this as a working hypothesis. We can then look for normal forms of qubits relative to the action of local transformations. The most naive method is to look at orbits of $G$ in projective $N-1$ space $\left(\mathbb{P}\left(H_{n}\right)\right)$. A homogeneous function on $H_{n}$ is said to be a measure of entanglement if it is constant on each orbit of the local transformations. It is convenient to restrict our attention to complex valued polynomials on $H_{n}$ (looked upon as a real vector space of dimension $2 N$ ) which we shall do. Using the observations in [M-W1] one can show that if $v, w$ are unit vectors in $H_{n}$ and if $|f(v)|=|f(w)|$ for all measures of entanglement then $v$ and $w$ are in the same orbit of $G$ in $\mathbb{P}\left(H_{n}\right)$.

The first question is how many such measures exist in each degree? It is clear that the measures of entanglement in $n$-qubits of degree $k$ form a vector space over $\mathbb{C}$ of finite dimension, $d_{n, k}$. The set of all such dimensions is encapsulated in the formal series

$$
h_{n}(q)=\sum_{k=0}^{\infty} d_{n, k} q^{k} .
$$

This is called the Hilbert series of measures of entanglement in $n$-qubits. For $n=2$ this series is easily computed and is just

$$
\frac{1}{\left(1-q^{2}\right)^{3}} \text {. }
$$


For $n=3$ the problem is much more difficult ([M-W1], Markus Grasl ). The series in this case is

$$
\frac{\left(1+q^{4}\right)\left(1+q^{4}+q^{8}\right)}{\left(1-q^{2}\right)\left(1-q^{4}\right)^{5}\left(1-q^{6}\right)}
$$

In the theses case we can write down explicit generators predicted by the series for $n=2$ three elements of degree 2 that generate and in the second case 9 generators with 1 of degree 2 (the Hilbert space norm squared), 7 of degree 4 and one of degree 6.

The purpose of this article is to explain why the Hilbert series in the case $n=4$ is given by

$$
\frac{P(q)}{\left(1-q^{2}\right)^{3}\left(1-q^{4}\right)^{11}\left(1-q^{6}\right)^{6}}
$$

with

$$
\begin{aligned}
& P(q)=. \\
& 1+3 q^{4}+20 q^{6}+76 q^{8}+219 q^{10}+654 q^{12}+ \\
& 1539 q^{14}+3119 q^{16}+5660 q^{18}+9157 q^{20}+ \\
& 12876 q^{22}+16177 q^{24}+18275 q^{26}+ \\
& 18275 q^{28}+16177 q^{30}+12876 q^{32}+ \\
& 9157 q^{34}+5660 q^{36}+3119 q^{38}+1539 q^{40}+ \\
& 654 q^{42}+219 q^{44}+76 q^{46}+20 q^{48}+3 q^{50}+q^{54}
\end{aligned}
$$

As the reader will see that our justification of this formula is difficult, partially done by computer and partially an outgrowth of serendipity. The determination of generators for the measures of entanglement will have to wait for later papers. However, the formula clearly demonstrates that the determination of all measures of entanglement is not a good idea for 4 qubits or more. To our mind a more reasonable problem is to find specific measures of entanglement that can help in the analysis of quantum algorithms (one such can be found in [M-W2]). We should point out that by doing explict residue integrals Markus Grasl and Martin Reotteler have derived the analogous formula for the action of the direct product of 4 copies of $U(2)$. (Using our methods we have derived the same formula which you can find without proof at the end of this paper. They also have a conjectured formula for the bigraded Hilbert series for 4 copies of $S U(2)$ which certainly can be done by our methods but we have not made the check as yet.)

The calculations we will be describing turn out to be possible since the situation of 4 qubits fits into the formulation of Kostant-Rallis [K-R]. It is for this reason there is a sizable section on symmetric pairs.

This formula was initially determined using a false ansatz (the falsity pointed out to the author by Hanspeter Kraft) which will be explained in the last section. The various methods used in reducing the degrees that should appear in a "true" denominator in such a formula involve results of Knop and Hochster-Roberts, ideas of Popov, Kraft,and Derksen and forthcoming joint work of the author with Kraft. The calculation of the dimensions $d_{4, k}$ for $k \leq 134$ was aided in its early stages by a marvelous formula of Thomas Enright which allowed us to give an independent check of the correctness of the $\mathrm{C}$ code eventually used in the full calculation. The actual calculation was carried out using an array of computers that was established with the help of the NSF to do large scale symbolic calculations (the Groebner 
array). We would like to thank Enright for the formula alluded to above (which is an outgrowth of his joint work with Jeb Willenbring, [E-W]) and Hanspeter Kraft for numerous discussions, theorems and counterexamples.

\section{Some Generalities.}

We first recall some material from [M-W1]. Let $G$ be the $n$-fold product of $S U(2)$ with itself (as in the introduction) acting on $H_{n}=\otimes^{n} \mathbb{C}^{2}$ by the tensor product action. If we use the basis of bit strings as in the introduction then if $v \in H_{n}$ we have $\left(N=2^{n}\right)$

$$
v=\sum_{j=0}^{N-1} x_{j}|j\rangle
$$

a complex valued polynomial, $f$, on $H_{n}$ (as a real vector space) is given by

$$
f(v)=g\left(x_{0}, \ldots, x_{N-1}, \bar{x}_{0}, \ldots, \bar{x}_{N-1}\right)
$$

with $g$ a polynomial in $2 N$ variables. Since the conjugate of a unitary matrix is its transpose inverse we see that the dimension of the space of measures of entanglement of degree $k$ is equal to the dimension of the space of homogenous invariants of $G$ acting on the homogeneous polynomials on $H_{n} \times H_{n}^{*}$ with $G$ acting on the second factor by the contragradient representation. We note that as representations of $G, H_{n}$ and $H_{n}^{*}$ are unitarily equivalent. Thus the problem of calculating the numbers $d_{n, j}$ in the introduction is reduced to calculating the dimension of the space of homogeneous (complex analytic) polynomials of degree $k$ on $H_{n} \times H_{n}$ that are invariant under the diagonal action of $G$. Since the polynomials are complex analytic the invariance extends to the action of the complexified group which is the $n$-fold copies of $S L(2, \mathbb{C})$. For the remainder of this paper we will work in the context of complex groups acting on complex vector spaces and considering complex analytic polynomials that are invariant under the groups. With this in mind we begin in earnest.

Let $V$ be a finite dimensional vector space over $\mathbb{C}$ and let $G$ be a Zariski closed reductive subgroup of $G L(V)$. Let $\mathcal{O}(V)_{+}^{G}$ denote the space of $G$-invariant polynomials, $f$, on $V$ such that $f(0)=0$. Let $\mathcal{N}(V)$ be the null cone of $V$ (that is, $\left.\mathcal{N}(V)=\left\{v \in V \mid f(v)=0, f \in \mathcal{O}(V)_{+}^{G}\right\}\right)$. The theorem of Hochster and Roberts [H$\mathrm{R}]$ implies that if $u_{1}, \ldots, u_{r}$ are algebraically independent, homogeneous, elements of $\mathcal{O}(V)_{+}^{G}$ such that the ideal in $\mathcal{O}(V)^{G}$ that they generate contains a power of $\mathcal{O}(V)_{+}^{G}$ (i.e. a homogeneous system of parameters) then as a $\mathbb{C}\left[u_{1}, \ldots, u_{r}\right]$-module $\mathcal{O}(V)^{G}$ is free on a finite number of homogeneous generators. Let $u_{1}, \ldots, u_{r}$ be a homogeneous system of parameters for $\mathcal{O}(V)^{G}$ with $d_{i}=\operatorname{deg} u_{i}$ for $i=1, . ., r$. Then the theorem asserts that the Hilbert series of $\mathcal{O}(V)^{G}$ is of the form

$$
\frac{P(q)}{\left(1-q^{d_{1}}\right)\left(1-q^{d_{2}}\right) \cdots\left(1-q^{d_{r}}\right)}
$$

with $P(q)$ a polynomial with non-negative integral coefficients. A theorem of Knop $[\mathrm{K}]$ implies that

$$
\sum_{i=1}^{r} d_{i}-\operatorname{deg} P(q) \geq \operatorname{dim} V .
$$

In fact, in most interesting cases (including the ones studied in this paper) the inequality can be replace by equality. This inequality implies that if we know the degrees of a system of parameters then we have an upper bound on the degree of 
the corresponding numerator of the Hilbert series and so the determination of the Hilbert series "only" involves knowing $\operatorname{dim} \mathcal{O}^{j}(V)^{G}$ for

$$
0 \leq j \leq \sum_{i=1}^{r} d_{i}-\operatorname{dim} V .
$$

This observation combined with Hilbert's criterion is the basis of all modern "effective" computations of rings of invariants.[P1],[P2],[D-K],[D].

We will describe Hilbert's criterion by combining two statements that are each separately called a Hilbert criterion.

1) $v \in \mathcal{N}(V)$ if and only if there exists an algebraic group homomorphism, $\phi$, of $G L(1, \mathbb{C})=\mathbb{C}^{\times}=\mathbb{C}-\{0\}$ into $G$ such that $\lim _{z \rightarrow 0} \phi(z) v=0$.

2) A set algebraically independent homogeneous elements, $\left\{u_{1}, \ldots, u_{r}\right\}$ in $\mathcal{O}(V)_{+}^{G}$ is a system of parameters if and only if $\mathcal{N}(V)=\left\{v \in V \mid u_{i}(v)=0, i=1, \ldots, r\right\}$.

The idea here is that it is usually easier to determine the structure of the null cone than that of the ring of invariants. Obviously, the goal is to find a system of parameters whose product has the lowest possible degree. Before we go on we give a simple consequence of 1) above.

Lemma 1. Let $G$ act on $V \times V$ diagonally if $(u, v) \in \mathcal{N}(V \times V)$ and if $W$ is the linear span of $u, v$ then $W \times W \subset \mathcal{N}(V \times V)$.

Proof. By the Hilbert criterion there exists $\phi: \mathbb{C}^{\times} \rightarrow G$ an algebraic homomorphism such that

$$
\lim _{z \rightarrow 0}(\phi(z) u, \phi(z) v)=0 .
$$

This implies that if $w \in W$ then $\lim _{z \rightarrow 0} \phi(z) w=0$. Thus if $x, y \in W$ then

$$
\lim _{z \rightarrow 0}(\phi(z) x, \phi(z) y)=0 \text {. }
$$

The Hochster-Roberts theorem is usually phrased as the assertion that $\mathcal{O}(V)^{G}$ is a Cohen-Macaulay ring. Thus the conditions in 1),2) above are a criterion for $u_{1}, \ldots, u_{r}$ to be a maximal regular sequence in $\mathcal{O}(V)^{G}$. We will now derive some results that will help us in our quest to make the eventual computer calculations manageable. The first is easy and well known.

Lemma 2. If the set $u_{1}, \ldots, u_{r} \in \mathcal{O}(V)_{+}^{G}$ is a regular sequence in $\mathcal{O}(V)$ then it is a regular sequence in $\mathcal{O}(V)^{G}$.

Proof. Suppose that $g \in \mathcal{O}(V)^{G}$ and

$$
g u_{i+1} \in \sum_{j \leq i} \mathcal{O}(V)^{G} u_{j}
$$

Then by the definition of regular sequence there exist elements $f_{j} \in \mathcal{O}(V)$ such that

$$
g=f_{1} u_{1}+\ldots+f_{i} u_{i} .
$$

If we apply the Reynold's operator(the $G$-invariant projection onto the invariants), $R$, we have

$$
g=R\left(f_{1}\right) u_{1}+\ldots+R\left(f_{i}\right) u_{i} .
$$


The next result is only slightly harder. In the rest of the section $G$ will not play a role.

Lemma 3. Assume that $u_{1}, \ldots, u_{l}$ is a regular sequence $\mathcal{O}(V)$ such that the ideal $I=\sum \mathcal{O}(V) u_{i}$ is a radical ideal. If $f \in \mathcal{O}(V)$ is such that it is non-zero on every irreducible component of $X\left(u_{1}, \ldots, u_{l}\right)=\left\{v \in V \mid u_{i}(v)=0, i=1, \ldots, l\right\}$. Then $u_{1}, \ldots, u_{l}, f$ is a regular sequence in $\mathcal{O}(V)$.

Proof. Let $X=X\left(u_{1}, \ldots, u_{l}\right)=\cup_{j=1}^{d} X_{j}$ with the $X_{j}$ the irreducible components of $X$. If

$$
g f \in \sum_{j \leq l} \mathcal{O}(V) u_{j}
$$

then $g f_{\mid X_{j}}=0$ for all $j=1, \ldots, d$. Since $f_{\mid X_{j}} \neq 0$ for all $j$ this implies that $g_{\mid X_{j}}=0$ for all $j$ (since each $X_{j}$ is irreducible). Hence the nullstellensatz implies that $g$ is in the radical of $I$. Now $g \in I$ since $I$ is assumed to be a radical ideal. This is the assertion of the lemma.

We will also use the following standard variant of the Noether normalization.

Lemma 4. Let $R$ be a graded commutative algebra over $\mathbb{C}$ with homogenous components $R^{j} j=0,1, \ldots$ and $\operatorname{dim} R^{j}<\infty, R^{0}=\mathbb{C} 1$. Assume that $j_{o}$ is such that $R$ is generated (as a ring) by $R^{j_{o}}$ and $R^{0}$. Then there exist algebraically independent elements $u_{1}, \ldots, u_{r}$ in $R^{j_{o}}$ such that $R$ is integral over $\mathbb{C}\left[u_{1}, \ldots, u_{r}\right]$.

We will sketch the argument that proves this result. Let $v_{1}, \ldots, v_{m}$ be a basis of $R^{j_{0}}$. If $v_{1}, \ldots, v_{m}$ are algebraically independent then we are done. Otherwise there is $f \neq 0$ with $f \in \mathbb{C}\left[x_{1}, \ldots, x_{m}\right]\left(x_{1}, \ldots, x_{m}\right.$ indeterminates $)$ and $f\left(v_{1}, \ldots, v_{m}\right)=$ 0 . Let $f_{k}$ denote the $k$-th homogenous component of $f$. Then since the $v_{i}$ are homogeneous of the same degree we have $f_{k}\left(v_{1}, \ldots, v_{m}\right)=0$ for all $k$. We may thus assume that $f$ is homogeneous of degree $d$. Let $t_{1}, \ldots, t_{m-1}$ be another set of indeterminates and put $u_{i}=v_{i}-t_{i} v_{m}, i=1, \ldots, m-1$ and $u_{m}=v_{m}$. Then $v_{i}=u_{i}+t_{i} v_{m}$ for $i=1, \ldots, m-1$. Hence if $i_{1}+\ldots+i_{m}=d$ then

$$
v_{1}^{i_{1}} \cdots v_{m}^{i_{m}}=t_{1}^{i_{1}} \cdots t_{m-1}^{i_{m-1}} v_{m}^{d}+h_{i_{1} \cdots i_{m}}\left(u_{1}, \ldots, u_{m-1}, t_{1}, \ldots, t_{m-1}, v_{m}\right)
$$

with the degree of $h_{i_{1} \cdots i_{m}}$ in $v_{m}$ strictly less that $d$. This implies that (after expanding $f$ into monomials we have

$$
0=\sum_{i<d} h_{i}\left(u_{1}, \ldots, u_{m-1}, t_{1}, \ldots, t_{m-1}\right) v_{m}^{i}+f\left(t_{1}, \ldots, t_{m-1}, 1\right) v_{m}^{d} .
$$

Since $f$ is homogeneous and non-zero $f\left(t_{1}, \ldots, t_{m-1}, 1\right)$ cannot be identically 0 . We choose $t_{1}, \ldots, t_{m-1}$ so that $f\left(t_{1}, \ldots, t_{m-1}, 1\right) \neq 0$. Hence $v_{m}$ is integral over the algebra, $S$, generated by $\mathbb{C} 1$ and $u_{1}, \ldots, u_{m-1}$. We replace $R$ by $S$ and apply the same argument $\left(S^{j_{o}}\right.$ has basis $\left.v_{1}, \ldots, v_{m-1}\right)$.

The next result is a standard "Cohen-Macaulay" argument.

Lemma 5. Let $R=\mathcal{O}(V)$ with $V$ a finite dimensional vector space over $\mathbb{C}$ and let $u_{1}, \ldots, u_{l}$ be algebraically independent homogeneous elements of $R$ such that $R$ is free over $\mathbb{C}\left[u_{1}, \ldots, u_{l}\right]$. Let $I$ be the ideal generated by $u_{1}, . ., u_{l}$ and let $S=R / I$. Let $v_{1}, \ldots, v_{m}$ be homogeneous elements such that if $\overline{v_{i}}=v_{i}+I$ then $\overline{v_{1}}, \ldots, \overline{v_{m}}$ are algebraically independent and $S$ is integral over $\mathbb{C}\left[\overline{v_{1}}, \ldots, \overline{v_{m}}\right]$. Then

1) $u_{1}, \ldots, u_{l}, v_{1}, \ldots, v_{m}$ are algebraically independent. 
2) $R$ is free as a $\mathbb{C}\left[u_{1}, \ldots, u_{l}, v_{1}, \ldots, v_{m}\right]$-module on a finite number of homogeneous elements.

Proof. It is standard ( $c f$. [Mat, Theorem 17.3]) that $R / I$ is Cohen-Macaulay. Our hypotheses imply that $\overline{v_{1}}, \ldots, \overline{v_{m}}$ is a system of parameters for $S$. Hence $m+l=$ $\operatorname{dim} V$. Thus $S$ is a free $\mathbb{C}\left[\overline{v_{1}}, \ldots, \overline{v_{m}}\right]$ module on homogenous generators $\overline{w_{1}}, \ldots, \overline{w_{n}}$. Let $w_{1}, \ldots, w_{n}$ be homogenous such that $\overline{w_{i}}=w_{i}+I$. It is easy to see that if $P$ is the ring generated by $u_{1}, \ldots, u_{l}, v_{1}, \ldots, v_{m}$ then $\sum P w_{i}=R$. Thus $R$ is integral over $P$ so $u_{1}, \ldots, u_{l}, v_{1}, \ldots, v_{m}$ are algebraically independent. The rest is equally easy.

Lemma 6. Let $X$ be an algebraic variety with $G$ acting algebraically only a finite number of $G$-orbits. Let $Y$ be a closed subvariety of $X$ then there exists $y \in Y$ such that $G y \cap Y$ is Zariski open in $Y$.

Proof. If there exists an open orbit $\mathcal{O}$ in $X$ such that $Y \cap \mathcal{O} \neq \emptyset$ then there exists $y \in Y$ such that $\mathcal{O}=G y$ and $G y \cap Y$ is open in $Y$. If there is no such open orbit then let $U_{1}$ be the union of the open orbits in $X$ then $X_{1}=X-U$ is Zariski closed and $G$-invariant with only a finite number of $G$-orbits. Now apply the same argument to $X_{1}$. After a finite number of steps we have our conclusion.

Lemma 7. Assume that $\mathcal{N}(V)$ has only a finite number of $G$-orbits. Let $W$ be a subspace of $V$ contained in $\mathcal{N}(V)$. Then there exists $w \in W$ such that $G w \cap W$ is Zariski open (and dense) in $W$. Furthermore, if $y \in W$ is such that $w$ and $y$ are linearly independent then $y \in \mathfrak{g} w$.

Proof. The first assertion is a direct consequence of the previous lemma. Thus if $s, t \in \mathbb{C}$ and if $(s, t)$ is sufficiently close to $(1,0)$ then $s w+t y \in G w$. Let $H=$ $\{g \in G \mid g w=w\}$. Then there exists a subspace $Z$ of $\mathfrak{g}$ such that $\mathfrak{g}=\operatorname{Lie}(H) \bigoplus Z$ a direct sum of vector spaces. There exits a neighborhood $U$ of 0 in $Z$ so that the map $z \longmapsto \exp (z) H$ is a holomorphic diffeomorphism of $U$ onto a neighborhood of the identity coset of $G / H$. We therefore see that there exists $\varepsilon>0$ such and a holomorphic map $z$ on $\{(s, t)|| s-1|| t \mid,<\varepsilon\}$ with values in $Z$ such that if $|s-1|,|t|<\varepsilon$ then

and $z(1,0)=0$. Now

$$
\exp (z(s, t)) w=s w+t y
$$

$$
\frac{\partial}{\partial t} \exp (z(s, t)) w_{\mid s=1, t=0}=y .
$$

On the other hand since the differential of the exponential map at 0 is the identity we see that

$$
\frac{\partial}{\partial t} \exp (z(s, t)) w_{\mid s=1, t=0}=\frac{\partial z}{\partial t}(1,0) w \in \mathfrak{g} w .
$$

\section{Applications to Certain symmetric pairs.}

We now come to the class of examples that are of critical importance to this paper. Let $\mathfrak{g}$ be a semi-simple Lie algebra over $\mathbb{C}$ and let $\theta$ be an involutive automorphism of $\mathfrak{g}$. We set $\mathfrak{k}=\{x \in \mathfrak{g} \mid \theta x=x\}$ and $\mathfrak{p}=\{x \in \mathfrak{g} \mid \theta x=-x\}$. We denote by $G$ the group of inner automorpisms of $\mathfrak{g}$ and by $K$ the identity component of the centralizer of $\theta$ in $G$. For the rest of the section, $\mathfrak{p} \times \mathfrak{p}$ will play the role of $V$ 
and $K$ acting diagonally on $\mathfrak{p} \times \mathfrak{p}$ will play the role of $G$. We will assume that there is a Cartan subalgebra, $\mathfrak{h}$, of $\mathfrak{g}$ contained in $\mathfrak{k}$. Let $H$ denote the corresponding Cartan subgroup of $K$. Let $\Phi$ be the root system of $\mathfrak{g}$ with respect to $\mathfrak{h}$. Let $\Phi_{k}$ be the subset of roots of $\mathfrak{k}$ with respect to $\mathfrak{h}$ and $\Phi_{n}=\Phi-\Phi_{k}$. We fix a system of positive roots, $\Phi_{k}^{+}$, for $\Phi_{k}$. Let $\mathcal{P}$ be the set of all positive toots systems for $\Phi$ that contain $\Phi_{k}^{+}$. If $P \in \mathcal{P}$ then we set $P_{n}=P-\Phi_{k}^{+}$. If $\alpha \in P_{n}$ than the corresponding root space, $\mathfrak{g}_{\alpha}$, is contained in $\mathfrak{p}$. We set $\mathfrak{p}_{P}^{+}=\sum_{\alpha \in P_{n}} \mathfrak{g}_{\alpha}$. The following lemma is a special case of results that will appear in forthcoming work of Hanspeter Kraft and the author.

Lemma 8. Let $\mathfrak{p}^{m}$ denote the $m$ fold product of $\mathfrak{p}$ with itself with $K$ acting diagonally. Then

$$
\mathcal{N}\left(\mathfrak{p}^{m}\right)=\bigcup_{P \in \mathcal{P}} \operatorname{Ad}(K)\left(\mathfrak{p}_{P}^{+}\right)^{m} .
$$

Each of the spaces $\operatorname{Ad}(K)\left(\mathfrak{p}_{P}^{+}\right)^{m}$ is Zariski closed in $\mathcal{N}\left(\mathfrak{p}^{m}\right)$ and irreducible.

Proof. We will use Hilbert's criterion. Assume that $\phi: \mathbb{C}^{\times} \rightarrow K$ is an algebraic group homomorphism. Then there exists $k \in K$ such that $k \phi(z) k^{-1} \in H$ for all $z$. By choosing an appropriate element of the Weyl group of $K$ on $H$ we may also assume that $\alpha\left(k \phi(z) k^{-1}\right)=z^{n_{\alpha}}$ with $n_{\alpha}$ a non-negative integer. Set $\mu(z)=k \phi(z) k^{-1}$. In general if $\alpha \in \Phi$ then $\alpha(\mu(z))=z^{n_{\alpha}}$ with $n_{\alpha} \in \mathbb{Z}$. If $x=\left(x_{1}, \ldots, x_{m}\right), x_{i} \in \mathfrak{p}$ is such that

$$
\lim _{z \rightarrow 0} A d(\phi(z)) x=0
$$

then writing each $x_{i}$ in terms of its root space decomposition we see that $x_{i}=$ $\sum_{\alpha \in \Phi}\left(x_{i}\right)_{\alpha}$. The displayed condition implies that if $\left(x_{i}\right)_{\alpha} \neq 0$ then $n_{\alpha}>0$. Let $Q=\left\{\alpha \in \Phi \mid n_{\alpha}>0\right\}$. Then one can check that there is an element $P \in \mathcal{P}$ such that $Q \subset P$. This implies then decomposition asserted for the nullcone. Let $B_{k}$ be the Borel subgroup of $K$ corresponding to $\Phi_{k}^{+}$. Then we have a morphism of the vector bundle $K \times{ }^{B_{k}}\left(\mathfrak{p}_{P}^{+}\right)^{m}$ over the flag variety $K / B_{k}$ onto $A d(K)\left(\mathfrak{p}_{P}^{+}\right)^{m}$ given by $(k, x) \longmapsto A d(k) x$. Thus since the flag variety is projective and irreducible the last two assertions follow.

It is a theorem of Kostant and Rallis $[\mathrm{K}-\mathrm{R}]$ that $\mathcal{O}(\mathfrak{p})^{K}$ is a polynomial ring in homogeneous generators $u_{1}, \ldots, u_{l}$. In addition they proved that the ideal generated by $u_{1}, \ldots, u_{l}$ is a radical ideal and that $u_{1}, \ldots, u_{l}$ is a regular sequence in $\mathcal{O}(\mathfrak{p})$. If $x, y \in \mathfrak{p}$ we define $u_{i}^{x}(x, y)=u_{i}(x)$ and $u_{i}^{y}(x, y)=u_{i}(y)$. We note that $u_{1}^{x}, \ldots, u_{l}^{x}, u_{1}^{y}, \ldots, u_{l}^{y}$ play the same role for $G \times G$ as $u_{1}, \ldots, u_{l}$ do for $G$ thus the ideal in $\mathcal{O}(\mathfrak{p} \times \mathfrak{p})$ generated by $u_{1}^{x}, . ., u_{l}^{x}, u_{1}^{y}, \ldots, u_{l}^{y}$ is a radical ideal. We denote by $B$ the Killing form of $\mathfrak{g}$.

Lemma 9. Assume that $\mathfrak{g}$ is not isomorphic with a product of subalgebras of type $A_{1}$. The elements $u_{1}^{x}, \ldots, u_{l}^{x}, u_{1}^{y}, \ldots, u_{l}^{y}, B(x, y)$ form a regular sequence in $\mathcal{O}(\mathfrak{p} \times \mathfrak{p})$.

Proof. We assume that $\mathfrak{g}$ has at least one simple ideal that is not of type $A_{1}$. We consider the variety, $X$, of zeros of $u_{1}^{x}, \ldots, u_{l}^{x}, u_{1}^{y}, \ldots, u_{l}^{y}$. Then the previous lemma implies that the irreducible components are of the form $A d(K)\left(\mathfrak{p}_{P_{1}}^{+}\right) \times A d(K)\left(\mathfrak{p}_{P_{2}}^{+}\right)$ with $P_{1}, P_{2} \in \mathcal{P}$. 
We will show that if such a product is actually an irreducible component then $B_{\mid A d(K)\left(\mathfrak{p}_{P_{1}}^{+}\right) \times A d(K)\left(\mathfrak{p}_{P_{2}}^{+}\right)} \neq 0$. If $P_{1} \neq P_{2}$ both are in $\mathcal{P}$ there must be an element $\alpha \in P_{1}$ such that $-\alpha \in P_{2}$. Now $\alpha \in \Phi_{n}$ by the definition of $\mathcal{P}$. This implies that $B_{\mid \mathfrak{p}_{P_{1}}^{+} \times \mathfrak{p}_{P_{2}}^{+}} \neq 0$. We may assume that $P_{1}=P_{2}=P$.

Let $s_{o}$ be the longest element of the Weyl group of $K$ acting on $\mathfrak{h}$ relative to $\Phi_{k}^{+}$. We assert that if $s_{o} P_{n}=P_{n}$ then $\operatorname{Ad}(K)\left(\mathfrak{p}_{P}^{+}\right)=\mathfrak{p}_{P}^{+}$. Indeed it is enough to show that if $\alpha \in \Phi_{k}$ and if $\beta \in P_{n}$ is such that $\alpha+\beta \in \Phi$ then $\alpha+\beta \in P_{n}$. This is obvious if $\alpha \in \Phi_{k}^{+}$. If not then $\alpha \in s_{o} \Phi_{k}^{+}$. Thus (since $s_{o} P+P$ ) if $\beta \in P_{n}$ is such that $\alpha+\beta \in \Phi$ then $s_{o} \alpha+s_{o} \beta \in \Phi$ and hence $s_{o} \alpha+s_{o} \beta \in P_{n}$. Since $s_{o}^{-1} P_{n}=P_{n}$ we have $\alpha+\beta \in P_{n}$.

We therefore see that if $s_{o} P_{n}=P_{n}$ and if $\operatorname{Ad}(K)\left(\mathfrak{p}_{P}^{+}\right) \times \operatorname{Ad}(K)\left(\mathfrak{p}_{P}^{+}\right)$were an irreducible component of $X$ then $\mathfrak{p}_{P}^{+} \times \mathfrak{p}_{P}^{+}$would be an irreducible component of $X$. Since the results of Kostant and Rallis imply that $X$ is a complete intersection it is of pure dimension. This implies that every irreducible component of $X$ has dimension equal to $\operatorname{dim} \mathfrak{p}_{P}^{+}=\left|\Phi_{n}\right| / 2$. This can only happen if $\operatorname{Ad}(K)\left(\mathfrak{p}_{P_{1}}^{+}\right)=\mathfrak{p}_{P_{1}}^{+}$ for all $P_{1} \in \mathcal{P}$. We assert that this implies that $\mathfrak{g}$ is a product of simple factors of type $A_{1}$. Indeed, if $P \in \mathcal{P}$ then there must be a simple root, $\alpha$, of $P$ that is in $P_{n}$ it is easily seen that $s_{\alpha} P \in \mathcal{P}$ and that

$$
\left(s_{\alpha} P\right)_{n}=\left(P_{n}-\{\alpha\}\right) \cup\{-\alpha\} .
$$

If the simple factor of $\mathfrak{g}$ containing the $\alpha$ root space is not of type $A_{1}$ then there must be another simple root, $\gamma$, in $P$ such that $\alpha+\gamma \in \Phi$. If $\gamma \in \Phi_{k}$ then $-\alpha-\gamma \in \Phi$ hence in $\left(P_{n}-\{\alpha\}\right) \cup\{-\alpha\}$. Hence $-\alpha-\gamma \in P_{n}$. But then $(-\alpha-\gamma)+\gamma \in P_{n}$ which is ridiculous. If $\gamma \in P_{n}$ then $\alpha+\gamma \in \Phi_{k}^{+}$. Hence $\alpha-(\alpha+\gamma) \in P_{n}$ which is also impossible. We have finally shown that if $\mathfrak{g}$ is not a product of simple factors of type $A_{1}$ and if $A d(K)\left(\mathfrak{p}_{P}^{+}\right) \times A d(K)\left(\mathfrak{p}_{P}^{+}\right)$is an irreducible component of $X$ then $s_{o} P_{n} \neq P_{n}$. Let $k \in K$ be such that $A d(k)_{\mid \mathfrak{h}}=s_{o}$. Then there is $\alpha \in P_{n}$ with $-\alpha \in s_{o} P_{n}$ and so $B_{\mid\left(\mathfrak{p}_{P}^{+} \times A d(k)\left(\mathfrak{p}_{P}^{+}\right)\right.} \neq 0$. This completes the proof of the Lemma.

Remark 1. If $\mathfrak{g}=\mathfrak{s l}(2, \mathbb{C})$ with the involution $\theta(X)=-X^{T}$ then the assertion of the previous result is easily seen to be false.

The next result gives an alternate interpretation of Lemma 5. Actually this lemma is true without the assumption that the rank of $\mathfrak{k}$ equals the rank of $\mathfrak{g}$. A more general result of this type will appear in $[\mathrm{K}-\mathrm{W}]$.

Proposition 1. Assume (only) that $\mathfrak{g}$ is semi-simple and that $\theta$ is an involutive automorphism of $\mathfrak{g}$. If $(x, y) \in \mathfrak{p} \times \mathfrak{p}$ then $(x, y) \in \mathcal{N}(\mathfrak{p} \times \mathfrak{p})$ if and only if the Lie algebra generated by $x, y$ consists of nilpotent elements.

If $(x, y) \in \mathcal{N}(\mathfrak{p} \times \mathfrak{p})$ then there exists an algebraic homomorphism $\phi: \mathbb{C}^{\times} \rightarrow K$ such that $\lim _{z \rightarrow 0}(\phi(z) x, \phi(z) y)=0$. This implies that if $w$ is in the Lie algebra generated by $x, y$ then $\lim _{z \rightarrow 0} \phi(z) w=0$. We conclude that $w$ is nilpotent hence proving the necessity. Suppose that $x, y \in \mathfrak{p}$ generate a Lie subalgebra consisting of nilpotent elements. The the Lie subalgebra generated by $x, y$ is $\theta$-invariant and consists of nilpotent elements. To complete the proof of this proposition we will need the following lemma.. 
Lemma 10. Let $\mathfrak{v}$ be a Lie subalgebra of $\mathfrak{g}$ that is $\theta$-stable and consists of nilpotent elements. Then there exists a $\theta$-stable Borel subalgebra, $\mathfrak{b}$, of $\mathfrak{g}$ such that $\mathfrak{v}$ is contained in $[\mathfrak{b}, \mathfrak{b}]$ (the nilradical of $\mathfrak{b}$ ).

Proof. Let $\mathfrak{m}$ be the set of all $x \in \mathfrak{g}$ such that $[x, \mathfrak{v}] \subset \mathfrak{v}$. Then $\mathfrak{m}$ is a $\theta$-stable subalgebra of $\mathfrak{g}$. By $[\mathrm{T}]$ there is a Levi decomposition $\mathfrak{m}=\mathfrak{s} \bigoplus \mathfrak{r}$ with $\mathfrak{s}$ a semisimple Lie subalgebra and $\mathfrak{r}$ the radical of $\mathfrak{m}$. We note that $\mathfrak{v}$ is contained in the nilradical, $\mathfrak{n}$, of $\mathfrak{r}$. If $\mathfrak{s} \neq 0$ then it contains a $\theta$-stable Borel subalgebra with nilradical $\mathfrak{u}$. Thus $\mathfrak{u} \bigoplus \mathfrak{n}$ is a theta stable subalgebra of $\mathfrak{g}$ consisting of nilpotent elements. We can now repeat the argument above with $\mathfrak{v}$ replaced by $\mathfrak{u} \bigoplus \mathfrak{n}$. After a finite number of steps we would find that the corresponding normalizer is solvable and is the normalizer in $\mathfrak{g}$ of its nilradical. We assert that this $\theta$ stable solvable subalgebra, $\mathfrak{r}$, is a desired Borel subalgebra. Indeed, if $\mathfrak{n}$ is the nilradical of this Lie algebra then it is precisely the set of all nilpotent elements in $\mathfrak{r}$. Thus $\mathfrak{n}$ is the set of all nilpotent elements, $x$, in $\mathfrak{g}$ such that $[x, \mathfrak{n}] \subset \mathfrak{n}$. We assert that this implies that $\mathfrak{n}$ is maximal among the Lie subalgebras of $\mathfrak{g}$ that consist of nilpotent elements. Indeed, if there were a larger such algebra, $\mathfrak{u}$ then Engel's theorem implies that there would be an $x \in \mathfrak{u}, x \notin \mathfrak{n}$ such that the element $x+\mathfrak{n}$ in $\mathfrak{u} / \mathfrak{n}$ is annihilated by $\mathfrak{n}$. Thus $[x, \mathfrak{n}] \subset \mathfrak{n}$. But then $x \in \mathfrak{n}$. This contradiction implies the assertion and hence the Lemma.

We can now conclude the proof of the proposition. Let $\mathfrak{v}$ be the Lie algebra generated by $x, y$. Then by hypothesis the elements of $\mathfrak{v}$ are nilpotent. The previous lemma implies that there is a $\theta$-stable Borel subalgebra, $\mathfrak{b}$, of $\mathfrak{g}$ such that $\mathfrak{v} \subset \mathfrak{n}=[\mathfrak{b}, \mathfrak{b}]$. We can chose a $\theta$-stable Cartan subalgebra, $\mathfrak{h}$, in $\mathfrak{b}$. This is well known but for the benefit of the reader we will give a proof below. If $H \in \mathfrak{h}$ is the dual vector to the sum of the positive roots then $\theta H=H$. If $\phi(z)=\exp (z H) \in K$ then if $x \in \mathfrak{n}$ then $\lim _{z \rightarrow 0} A d(\phi(z)) x=0$. Thus in particular, Hilbert's criterion implies that $(x, y)$ is in the null cone of $\mathfrak{p} \times \mathfrak{p}$. The proposition is now proved.

We will now prove the assertion in the above proof about $\theta$-stable Borel subalgebras.

Lemma 11. Let $\mathfrak{b}$ be a $\theta$-stable Borel subalgebra of $\mathfrak{g}$ then $\mathfrak{b} \cap \mathfrak{k}$ is a Borel subalgebra of $\mathfrak{k}$. Furthermore, $\mathfrak{b}$ contains a $\theta$-stable Cartan subalgebra.

Proof. Fix $\mathfrak{b}_{k}$, a Borel subalgebra of $\mathfrak{k}$. Let $\mathcal{B}$ denote the (flag variety) of all Borel subalgebras of $\mathfrak{g}$. Then $\theta$ acts on $\mathcal{B}$ algebraically. Let $\mathcal{B}^{\theta}$ denote the set of $\theta$-fixed points (i.e. the $\theta$-stable Borel subalgebras). Then $\mathcal{B}^{\theta}$ is a projective $K$-variety. If $\mathfrak{b} \in \mathcal{B}^{\theta}$ let $n(\mathfrak{b})=\operatorname{dim} \mathfrak{b} \cap \mathfrak{k}$. Let $m$ be the minimum of the $n(\mathfrak{b})$ for $\mathfrak{b} \in \mathcal{B}^{\theta}$. Then the set of all $\mathfrak{b} \in \mathcal{B}^{\theta}$ with $n(\mathfrak{b})=m$ is Zariski closed. The Borel fixed point theorem implies that there is an element $\mathfrak{b}$ of $\mathcal{B}^{\theta}$ with $n(\mathfrak{b})=m$ and $\left[\mathfrak{b}_{k}, \mathfrak{b}\right] \subset \mathfrak{b}$. This implies in particular that $\mathfrak{b}_{k}+\mathfrak{b}$ is a subalgebra of $\mathfrak{g}$ with $\mathfrak{b}$ a normal subalgebra. Thus, $\mathfrak{b}$ is contained in the radical of $\mathfrak{b}_{k}+\mathfrak{b}$. Since $\mathfrak{b}$ is a maximal solvable subalgebra of $\mathfrak{g}$ this implies that $\mathfrak{b}$ is the radical. Hence $\mathfrak{b}_{k} / \mathfrak{b}_{k} \cap \mathfrak{b}$ is either 0 or semi-simple. The latter is impossible so $\mathfrak{b}_{k} \subset \mathfrak{b}$. In particular this implies that if $\mathfrak{b}_{1} \in \mathcal{B}^{\theta}$ then $n\left(\mathfrak{b}_{1}\right)=\operatorname{dim} \mathfrak{b}_{k}$. This implies the first assertion of the Lemma. To prove the second we may assume $\mathfrak{b}_{k}=\mathfrak{b} \cap \mathfrak{k}$ and that $\mathfrak{b}_{k}$ contains a regular semi-simple element, $h$, of $\mathfrak{g}$. The centralizer of $h$ in $\mathfrak{g}, \mathfrak{u}$, is reductive and the centralizer of $h$ 
in $\mathfrak{b}$ is a Borel subalgebra of $\mathfrak{u}$. Replacing $\mathfrak{g}$ by the commutator algebra of $\mathfrak{u}$ and $\theta$ by $\theta_{\mid \mathfrak{u}}$ the assertion follows by the obvious inductive argument.

We conclude this section by studying implications of Proposition 1.

Proposition 2. If $(x, y) \in \mathfrak{p} \times \mathfrak{p}$ let $\mathfrak{g}_{x, y}$ be the Lie algebra generated by $x, y$. If $x, y \in \mathcal{N}(\mathfrak{p})$ then $(x, y) \in \mathcal{N}(\mathfrak{p} \times \mathfrak{p})$ if and only if $B_{\mid \mathfrak{g}_{x, y} \cap \mathfrak{k}}=0$ if and only if $\mathfrak{g}_{x, y} \cap \mathfrak{k}$ consists of nilpotent elements.

Proof. The necessity of each of both of the above conditions follows from Hilbert's criterion. By $[\mathrm{T}]$ there exists a Levi decomposition $\mathfrak{g}_{x, y}=\mathfrak{s} \bigoplus \mathfrak{r}$ with $\mathfrak{s}$ a semisimple subalgebra of $\mathfrak{g}_{x, y}$ and $\theta$-invariant and $\mathfrak{r}$ the radical of $\mathfrak{g}_{x, y}$. If $\mathfrak{s} \neq 0$ then there must be a semi-simple element in $\mathfrak{k} \cap \mathfrak{s}$ since this algebra is reductive. Thus if $\mathfrak{g}_{x, y} \cap \mathfrak{k}$ consists of nilpotent elements then $\mathfrak{g}_{x, y}$ is solvable. We note that $B$ restricted to each simple factor of $\mathfrak{s}$ is non-zero (since each factor contains a standard triple $e, f, h$ with $[h, e]=2 e,[h, f]=-2 f,[e, f]=h$. In particular, $h \neq 0$ and $a d(h)$ has integral eigenvalues not all 0 so $B(h, h)>0$ ). If $\mathfrak{u}$ is a simple factor than either $\theta \mathfrak{u}=\mathfrak{u}$ or $\mathfrak{u} \cap \theta \mathfrak{u}=0$. We leave it to the reader to check that in the first case $B_{\mid \mathfrak{u} \cap \mathfrak{k}} \neq 0$. In the second (since $(\mathfrak{u}+\theta \mathfrak{u}) \cap \mathfrak{k}$ is isomorphic with $\mathfrak{u}$ ) we have $B_{\mid(\mathfrak{u}+\theta \mathfrak{u}) \cap \mathfrak{k}} \neq 0$. Thus we see that if $B_{\mid \mathfrak{g}_{x, y} \cap \mathfrak{k}}=0$ then $\mathfrak{g}_{x, y}$ is solvable. We now show that if $\mathfrak{g}_{x, y}$ is solvable then it consists of nilpotent elements. Now Engel's theorem implies that there is a basis of $\mathfrak{g}$ such that the matrix of $a d(z)$ is upper triangular relative to this basis for all $z \in \mathfrak{g}_{x, y}$. Since $x, y$ are nilpotent the diagonal entries of their matrix are 0 . Since by definition $\mathfrak{g}_{x, y}$ is generated by $x, y$ we see that $\mathfrak{g}_{x, y}$ consists of nilpotent elements.

We will now show how to use this result to find a defining set of equations for the nullcone. If $x, y \in \mathfrak{g}$. Then a Lie word in $x, y$ is a non-commutative monomial in the free Lie algebra in two generators with the generators specialized to $x, y$. If $w$ is such a monomial then we write $w(x, y)$ for the specialization. The free Lie algebra in two generators is naturally graded if we assign the degrees of the generators to both be 1 . Thus the monomials have a natural degree. If $w$ has degree $k$ and $w^{\prime}$ has degree $l$ then $x, y \rightarrow B\left(w(x, y), w^{\prime}(x, y)\right)$ is a polynomial in $x, y$ of degree $k+l$ (which, of course, could be 0 ).

The Proposition above can be interpreted as follows

Corollary 1. The nullcone $\mathcal{N}(\mathfrak{p} \times \mathfrak{p})$ is defined by

$$
u_{1}^{x}, \ldots, u_{l}^{x}, u_{1}^{y}, \ldots, u_{l}^{y} \cup\left\{B\left(w(x, y) . w^{\prime}(x, y)\right) \mid w, w^{\prime} \text { Lie words of even degree }\right\} .
$$

\section{First Reductions for 4 Qubits.}

We now come to the case of interest in this paper. We observe that if we take $\mathfrak{g}=\mathfrak{s} o(8, \mathbb{C})$ and $\theta(X)=J X J$ with $J$ the matrix

$$
\left[\begin{array}{cc}
I_{4} & 0 \\
0 & -I_{4}
\end{array}\right]
$$

with $I_{4}$ the $4 \times 4$ identity matrix then $\mathfrak{k}=\mathfrak{s o}(4, \mathbb{C}) \times \mathfrak{s} o(4, \mathbb{C})$ imbedded diagonally in $\mathfrak{g}$. We also not that $\mathfrak{p}$ is the space of all matrices of the form

$$
\left[\begin{array}{cc}
0 & X \\
-X^{T} & 0
\end{array}\right]
$$


with $X$ an arbitrary $4 \times 4$ matrix over $\mathbb{C}$. We will identify the matrix above with $X$ and thereby think of $\mathfrak{p}$ as the space $M_{4}(\mathbb{C})$ of $4 \times 4$ matrices over $\mathbb{C}$. If we consider the adjoint action of $S O(8, \mathbb{C})$ on its Lie algebra then the action of $K$ on $\mathfrak{p}$ is

$$
\left(k_{1}, k_{2}\right) X=k_{1} X k_{2}^{-1} .
$$

We note that there is a covering homomorphism, $\sigma$, of $S L(2, \mathbb{C}) \times S L(2, \mathbb{C}) \times$ $S L(2, \mathbb{C}) \times S L(2, \mathbb{C})$ onto $\mathfrak{s o}(4, \mathbb{C}) \times \mathfrak{s} o(4, \mathbb{C})$ and relative to this homomorphism the corresponding action of $S L(2, \mathbb{C}) \times S L(2, \mathbb{C}) \times S L(2, \mathbb{C}) \times S L(2, \mathbb{C})$ on $M_{4}(\mathbb{C})$ is equivalent with the tensor product action of $S L(2, \mathbb{C}) \times S L(2, \mathbb{C}) \times S L(2, \mathbb{C}) \times$ $S L(2, \mathbb{C})$ on $\mathbb{C}^{2} \otimes \mathbb{C}^{2} \otimes \mathbb{C}^{2} \otimes \mathbb{C}^{2}$. This implies that the results of the previous section apply directly to the case of 4-qubits.

Thus the Hilbert series of the invariants for the action of $K$ on $\mathfrak{p} \times \mathfrak{p}$. Is the Hilbert series of measures of entanglement for 4 qubits. Our key result is

Theorem 1. Let $\mathfrak{g}, \theta$ be as above. Then the nullcone of $\mathfrak{p} \times \mathfrak{p}$ is defined by invariants of degrees $2,4,6,8$ and 12 ..

Our proof of this result relies on calculations that were done using Mathematica. We will describe the high points of the calculation. So that a serious reader would have no trouble repeating our calculations. We start by observing that in this case the nullcone of $\mathfrak{p}$ has four irreducible components that are permuted by the obvious action of $S_{4}$ when $\mathfrak{p}$ is looked upon as $\mathbb{C}^{2} \otimes \mathbb{C}^{2} \otimes \mathbb{C}^{2} \otimes \mathbb{C}^{2}$. The reason for this is the general fact that if $\mathfrak{p}$ contains a Cartan subalgebra of $\mathfrak{g}$ (which is the case for the example in question) then if $P \in \mathcal{P}$ then $\operatorname{Ad}(K) \mathfrak{p}_{P}^{+}$is an irreducible component of $\mathcal{N}(\mathfrak{p})$ if and only if every simple root of $P$ is in $\Phi_{n}$. One can check that there are 4 such choices permuted transitively by the obvious action of $S_{4}$ on $\otimes^{4} \mathbb{C}^{2}$. We first observe that if we consider the polarizations of the elements of $\mathcal{O}(\mathfrak{p})^{K}$ then these are generated as an algebra by invariants of degrees 2,4 and $6\left(\mathcal{O}(\mathfrak{p})^{K}\right.$ is generated by 4 homogeneous elements of respective degrees $\left.2,4,4,6\right)$. The set of zeros of these polarizations is exactly the set of all $x, y \in \mathfrak{p}$ such that $a x+b y \in \mathcal{N}(\mathfrak{p})$ for all $a, b \in \mathbb{C}$. Thus we may assume this property and that $x$ is in one of the irreducible components. We note that Lemma 6 implies that we can thus assume that both $x, y$ are in the same irreducible component since affine 2 space is an irreducible variety. Our problem is to show that there is a choice of $k$ and $P$ such that $x, y \in A d(k) \mathfrak{p}_{P}^{+}$(here we can't assume that $P$ is one of the 4 singled out). To set up the necessary computation we will need to do a bit more preparation.

We note that in this case $\mathfrak{k}$ is isomorphic with a direct sum of 4 copies of $\mathfrak{s l}(2, \mathbb{C})$, that is $\mathfrak{k}=\mathfrak{k}_{1} \oplus \mathfrak{k}_{2} \oplus \mathfrak{k}_{3} \oplus \mathfrak{k}_{4}$. Let $B_{i}$ denote the Killing form of $\mathfrak{k}_{i}$. Then the condition that $x=x_{1}+x_{2}+x_{3}+x_{4}$ is nilpotent is that $B_{i}\left(x_{i}, x_{i}\right)=0$ for $i=1,2,3,4$. We also observe

I) If $x, y \in \mathfrak{p}$ and that $u, v$ is another spanning set for the span of $x, y$ then all linear combinations of Lie words in $x, y$ of degree $2 r$ are nilpotent if and only if this is so for $u, v$.

We will show that if $x, y \in \mathfrak{p}$ are such that $a x+b y \in \mathcal{N}(\mathfrak{p})$ for all $a, b \in \mathbb{C}$ then $(x, y) \in \mathcal{N}(\mathfrak{p} \times \mathfrak{p})$ if and only if all linear combinations of Lie words of degrees 2, 4 
and 6 are nilpotent. This combined with that fact that nilpotence in $\mathfrak{k}$ is defined by 4 quadratic equations will prove the theorem. We note that the observation I) above combined with Lemma 7 allows us to assume that $y \in a d(\mathfrak{k}) x$ and by the observed symmetry we may assume that $x$ it is in one of the given 4 sets $A d(k) \mathfrak{p}_{P}^{+}$. The actual computation progresses as follows. We chose for $x$ a representative of a $K$-orbit in $\mathfrak{p}_{P}^{+}$. Then look at all $y \in a d(\mathfrak{k}) x$ such that $a x+b y \in \mathcal{N}(\mathfrak{p})$ for all $a, b \in \mathbb{C}$. We calculate the condition that certain Lie words of degrees $2,4,6$ are nilpotent and conclude that this implies that $(x, y) \in \mathfrak{p}_{P}^{+} \times \mathfrak{p}_{P}^{+}$. The actual calculation was done using Mathematica and a notebook for this calculation can be found on my website. There is a hyperlink there to Hilbert series 4-qubits computations. The first file in that link is a mathematica notebook: DetailsTheorem1.nb. This notebook calls newtry.m (a text file containing mathematica code). This code sets up the qubits as polynomials in $x[i, j], i=0,1,2,3$ and $j=0,1$ that are of total degree 4 and the monomials have exactly one of $x[i, 0]$ or $x[i, 1]$ with degree 1 . Thus

$$
|0110\rangle \rightarrow x[0,0] x[1,1] x[2,1] x[3,0] .
$$

The general element of $\mathfrak{k}$ is represented by and array

$$
y=\{a[0], b[0], c[0], a[1], b[1], c[1], a[2], b[2], c[2], a[3], b[3], c[3]\}
$$

representing 4 marices the $i$-th being

$$
\left[\begin{array}{cc}
a[i] & b[i] \\
c[i] & -a[i]
\end{array}\right] .
$$

The calculations choose a state in one orbit for, $z, K$ and show that if the Lie words of $z$ and $y \cdot z$ if the span of $z$ and $y . z$ conists of nilpotent elments and the Lie words of degrees 2, 3, 4, 6 are nilpotent then $z, y . z$ are in $A d(k) \mathfrak{p}_{P}^{+}$with the same $k$ and $P$.

\section{The Calculation of the Hilbert series.}

We are ready to describe the last steps in the calculation of the Hilbert series for the measures of entanglement for 4 qubits that is the Hilbert series for

$R=\mathcal{O}\left(\mathbb{C}^{2} \otimes \mathbb{C}^{2} \otimes \mathbb{C}^{2} \otimes \mathbb{C}^{2} \bigoplus \mathbb{C}^{2} \otimes \mathbb{C}^{2} \otimes \mathbb{C}^{2} \otimes \mathbb{C}^{2}\right)^{S L(2, \mathbb{C}) \times S L(2, \mathbb{C}) \times S L(2, \mathbb{C}) \times S L(2, \mathbb{C})}$

where $S L(2, \mathbb{C}) \times S L(2, \mathbb{C}) \times S L(2, \mathbb{C}) \times S L(2, \mathbb{C})$ acts on $V=\mathbb{C}^{2} \otimes \mathbb{C}^{2} \otimes \mathbb{C}^{2} \otimes \mathbb{C}^{2} \bigoplus \mathbb{C}^{2}$ by the tensor product action and on the two copies diagonally. Since $S L(2, \mathbb{C}) \times$ $S L(2, \mathbb{C}) \times S L(2, \mathbb{C}) \times S L(2, \mathbb{C})$ is semi-simple the indicated ring of invariants is a Gorenstein ring $[\mathrm{M}]$ which in this context implies that the Hilbert series is of the form

$$
\frac{P(q)}{\left(1-q^{d_{1}}\right)\left(1-q^{d_{2}}\right) \cdots\left(1-q^{d_{r}}\right)}
$$

with $d_{1}, \ldots, d_{r}$ the degrees of a homogeneous system of parameters for this ring of invariants with

$$
P(q)=1+a_{1} q+a_{2} q^{2}+\ldots+a_{d} q^{d}
$$

and $a_{j}=a_{d-j}$ for $j=0, \ldots, d$. That is $P(q)$ is palindromic.

If we apply Lemmas 9,5 , Hilbert's criterion 2 ) and Theorem 1 we see that if

$$
I=\sum R u_{i}^{x}+\sum R u_{i}^{y}+R B
$$


then $R / I$ has a system of parameters consisting of homogeneous elements of degree 24(the LCM of 2,4,6,8,12). A system of parameters of $R$ must consist of 20 elements ( $2 \operatorname{dim} V-\operatorname{dim} K=32-12$ ). Thus the Hilbert series can be written in the form

$$
\frac{P(q)}{\left(1-q^{2}\right)^{3}\left(1-q^{4}\right)^{4}\left(1-q^{6}\right)^{2}\left(1-q^{24}\right)^{11}}
$$

and by Knop's result $P(q)$ is of degree at most $24 \cdot 11+2 \cdot 6+4 \cdot 4+3 \cdot 2-32=266$ and in addition it must be palindromic. We note that it is clear that the homogeneous invariants must be of even degrees. This implies that if we find a the first 132 terms of $P(q)$ and if there is no duplication among the even ones then we can complete $P(q)$ by palindromy. This is not exactly what we did but the calculations that we did could be interpreted in this way. What we actually did was observe that the number of polarizations of $u_{1}^{x}, u_{2}^{x}, u_{3}^{x}, u_{4}^{x}$ is $3+2 \cdot 5+7=20$. We then guessed that these polarizations form a system of parameters for $R$. As it turns out that this is false (as Hanspeter Kraft pointed out to us). However, assuming this we hypothesized that the Hilbert series was given by

$$
\frac{U(q)}{\left(1-q^{2}\right)^{3}\left(1-q^{4}\right)^{10}\left(1-q^{6}\right)^{7}}
$$

with $U(q)$ of degree at most 56 (in light of Knop's result). We calculated the dimensions of the homogeneous elements of degree $j$ for $j=0,2, \ldots, 56$. We then solved for the coefficients of $U(q)$ that would give the same Taylor series to degree 56 and found that we had a palindrome. The outcome for $U(q)$ was

$$
\begin{gathered}
1+4 q^{4}+19 q^{6}+80 q^{8}+235 q^{10}+714 q^{12}+ \\
1698 q^{14}+3614 q^{16}+6704 q^{18}+11232 q^{20}+ \\
16461 q^{22}+21749 q^{24}+25579 q^{26}+27148 q^{28}+ \\
25579 q^{30}+21749 q^{32}+16461 q^{34}+11232 q^{36}+ \\
6704 q^{38}+3614 q^{40}+1698 q^{42}+714 q^{44}+ \\
235 q^{46}+80 q^{48}+19 q^{50}+4 q^{52}+q^{56}
\end{gathered}
$$

(It can be checked that the rational function here agrees with that in the introduction). We then checked up to 80 and found that the $U(q)$ calculated using the first 56 degrees gave the correct answer to degree 80 . At this point we exhausted every computer algebra system that we had worked with (including the low level system Groebner developed by Matt Clegg and the author) due to the immense amounts of memory necessary for these calculations. At this point the author wrote some special purpose $C$ code (which can be found in my website under Hilbert series 4-qubits computations) it actually calculates the dimensions of the homogeneous invariants of $\mathcal{O}\left(\otimes^{4} \mathbb{C}^{2} \oplus \otimes^{4} \mathbb{C}^{2}\right)^{K} /\left(\mathcal{O}\left(\otimes^{4} \mathbb{C}^{2} \oplus \otimes^{4} \mathbb{C}^{2}\right)^{K} u_{2}^{x}+\mathcal{O}\left(\otimes^{4} \mathbb{C}^{2} \oplus \otimes^{4} \mathbb{C}^{2}\right)^{K} u_{2}^{y}\right)$ with $u_{2}$ of degree 4 . The program calculated the degrees $82,84, \ldots, 134$ and found that they jibed with the hypothesis which one can check this by compiling the code and comparing the output with the coefficients of the Taylor series of the rational function

$$
\frac{U(q)}{\left(1-q^{2}\right)^{3}\left(1-q^{4}\right)^{8}\left(1-q^{6}\right)^{7}}
$$

Hence the theory we have developed implies that even though the initial guess about a system of parameters was false the answer it led to was correct. The 36 numbers that needed to be calculated were parcelled out to the Groebner array ( 8 pentium III's each accessing 1 gigabyte of RAM and 3 gigabytes of virtual memory). 
The $\mathrm{C}$ code observes that we can look at $\otimes^{4} \mathbb{C}^{2}$ as $\left(\otimes^{2} \mathbb{C}^{2}\right) \otimes\left(\otimes^{2} \mathbb{C}^{2}\right)$. We use the standard decomposition (usually called $G L(n), G L(n)$ Howe duality) of $\mathcal{O}\left(\mathbb{C}^{4} \otimes \mathbb{C}^{4}\right)$ as a $G L(4) \times G L(4)$ module as a direct sum

$$
\bigoplus\left(F^{\lambda}\right)^{*} \otimes\left(F^{\lambda}\right)^{*}
$$

the sum over all partitions $\left(\lambda_{1}, \lambda_{2}, \lambda_{3}, \lambda_{4}\right)$ with $\lambda_{1} \geq \lambda_{2} \geq \lambda_{3} \geq \lambda_{4} \geq 0$. We look upon the action of $S L(2, \mathbb{C}) \times S L(2, \mathbb{C})$ on $\otimes^{2} \mathbb{C}^{2}$ as $S O(4, \mathbb{C})$ acting on $\mathbb{C}^{4}$. The program branches the $F^{\lambda}$ with $\lambda_{4}=0$ from $S L(4, \mathbb{C})$ to $S O(4, \mathbb{C}) . F^{\lambda}=$ $\operatorname{det}^{\lambda_{4}} F^{\lambda^{\prime}}$ with $\lambda^{\prime}=\lambda-\lambda_{4}(1,1,1,1)$. This is the reason why we dropped 2 of the invariants of basic degree 4 . This is used to calculate the graded multiplicities in the appropriate degrees for one copy of 4 qubits. The program then uses the fact that the dimensions of the graded components of the invariants for two copies are easily calculated from the knowledge of the full graded decomposition in one copy.

\section{The Hilbert series for the CASE of $U(2) \times U(2) \times U(2) \times U(2)$.}

In this section we will give a formula without proof of the Hilbert series for the real polynomial invariants of the action of 4 copies of $U(2)$ on $\bigotimes^{4} \mathbb{C}^{2}$. One notes that the basic distinction is that we have a copy of $\mathbb{C}^{\times}$acting on the two copies of $\otimes^{4} \mathbb{C}^{2}$ with action by multiplictaion by $z \in \mathbb{C}^{\times}$on the first copy and by $z^{-1}$. Thus we look at the invariants of this action of $\mathbb{C}^{\times}$in the invariants of 4 copies of $S L(2, \mathbb{C})$ acting diagonally on two copies of $\otimes^{4} \mathbb{C}^{2}$. The Hilbert series is

$$
\frac{R(q)}{\left(1-q^{2}\right)\left(1-q^{4}\right)^{7}\left(1-q^{6}\right)^{6}\left(1-q^{8}\right)^{4}\left(1-q^{10}\right)}
$$

with $R(q)$ given by

$$
\begin{aligned}
& 1+6 q^{6}+46 q^{8}+110 q^{10}+344 q^{12}+844 q^{14} \\
& 2154 q^{16}+4606 q^{18}+9397 q^{20}+16848 q^{22}+ \\
& 28747 q^{24}+44580 q^{26}+65366 q^{28}+88036 q^{30}+ \\
& 111909 q^{32}+131368 q^{34}+145676 q^{36}+149860 q^{38} \\
& +145676 q^{40}+131368 q^{42}+111909 q^{44}+ \\
& 88036 q^{46}+65366 q^{48}+44580 q^{50}+28747 q^{52}+ \\
& 16848 q^{54}+9397 q^{56}+4606 q^{58}+2154 q^{60}+ \\
& 844 q^{62}+344 q^{64}+110 q^{66}+46 q^{68}+6 q^{70}+q^{76} .
\end{aligned}
$$

Notice that it is palendromic. This property is definitly not guaranteed by the theory. Also notice the 10 in the denomiator. From our prospective this causes a major increase in the computer calculations.

\section{REFERENCES}

[D] Harm Derkesen, Polynomial bounds for rings of invariants, Proc. Amer. Math. Soc. (129)2001,955-963.

[D-K] Harm Derksen and Hanspeter Kraft, Constructive invariant theory, Algèbra non commutative, groups quantiques et invariants (Reims,1995),221-244. Sémin. Congr. 2,Soc. Math. France.

[E-W] Thomas Enright and Jeb Willenbring, To appear Annals Math. 
[H-R] M. Hochster and J. Roberts, Rings of invariants of reductive groups acting on regular rings are Cohen-Macaulay, Adv. Math. 13 (1974) 115-175.

[K] F. Knop, Der kanonische Modul eines Invariantenringes, J. Algebra 127(1989),4054

[K-R] B. Kostant and S. Rallis, Orbits and Lie group representations associated with symmetric spaces, Amer. J. Math.,93(1971),753-809.

[K-W] Hanspeter Kraft and Nolan Wallach, (Provisional title) Remarks on the nullcone for diagonal actions, to appear.

[Mat] Hideyuki Matsumura, Commutative ring theory, Cambridge studies in advanced mathematics 8, Cambridgre University Press, Cambridge, 1986.

[M] M. Pavaman Murty, A note on factorial rings, Arch. Math. 15 (1964), 418-420.

[M-W1] David Meyer and Nolan Wallach, Invariants for multiple qubits: the case of 3 qubits, Mathematics of quantum computing, Computational Mathematics Series, 77-98, Chapman\&Hall/CRC, 2002.

[M-W2] David Meyer and Nolan Wallach,Global entanglement in multiparticle systems. Quantum information theory. J. Math. Phys. 43 (2002), no. 9, 4273-4278.

[P1] V. Popov, Constructive invariant theory, Astérisque 87-88(1981),303-334.

[P2] V. Popov, Constructive theory of invariants, Math. USSR Izvest. 10(1982),359376 .

[T] Earl J. Taft, Invariant Levi factors, Michigan Math J. 9(1962),65-68.

Department of Mathematics, UCSD

E-mail address: nwallach@ucsd.edu

$U R L:$ http://math.ucsd.edu/ nwallach 